\title{
An individually-controlled multi-tined expandable electrode using active-cannula-based shape morphing for on-demand conformal radiofrequency ablation lesions
}

\author{
Zhiping Chai ${ }^{1}$, Liangxiong $\mathrm{Lyu}^{1}$, Menghao $\mathrm{Pu}^{1}$, Huageng Liang ${ }^{1}$, Han Ding $^{1}$, and Zhigang \\ $\mathrm{Wu}^{1}$
}

${ }^{1}$ Affiliation not available

January 26, 2022

\begin{abstract}
Being minimally invasive and highly effective, radiofrequency ablation (RFA) is widely used for small size malignant tumors treatment. However, in clinical practice, a large number of tumors are found in irregular shape, while the current RFA devices are hard to control their morphologic appearance of RFA lesions on demand, which usually ends up excessively ablating the tissues and often brings excessively irreversible damage to the organs' functions. Here, we introduce active cannulas for each of individually-controlled sub-electrodes to achieve an on-demand shape morphing and thus conformal RFA lesion. The shape as well as the length of inserted sub-electrode can be precisely controlled by tuning the expanded length of the active stylet and relative position of the active cannula. Furthermore, owing to independent movement and energy control of each subelectrodes, our electrode is shown to be not only efficient enough to accomplish accurate trajectory to target tissue in a single insertion, but also adaptive enough to ablate target tissues with diverse morphologic appearances and locations. Potentially, our RFA electrode is a better choice in the future clinical practice for minimally invasive treatments of malignant tumors of which preferred treatment is conformal ablation.

Corresponding author(s) Email: zgwu@hust.edu.cn
\end{abstract}

\section{ToC Figure}




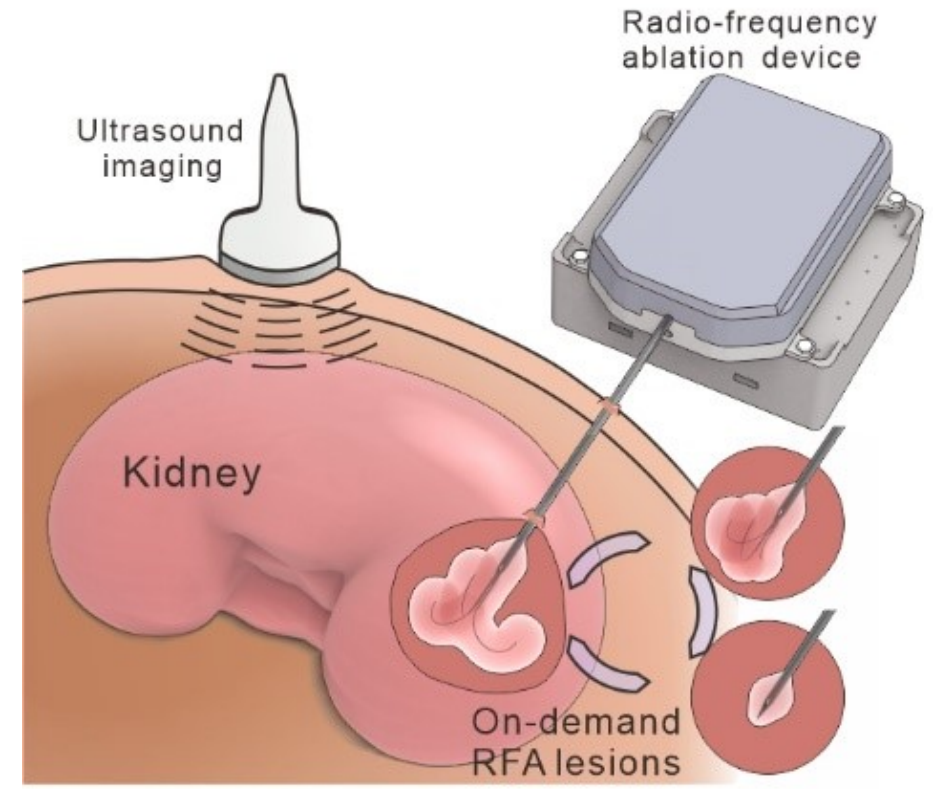

Figure 1: ToC Figure. An individual controlled multi-tined expandable electrode is presented here for ondemand conformal ablation of target tissues. The individually-controlled sub-electrodes of which the shape can be morphed by tuning the relative position of pre-curved stylets and stiff cannulas. With rational energy input and three sub-electrodes, our electrode can achieve three-dimensional on-demand conformal ablation efficiently.

\section{Introduction}

As a leading cause of mortality worldwide, tens of millions of people suffer from various cancers each year and the number is still ever-increasing. (Ormel et al., 2018; Sung et al., 2021; Bray et al., 2021)Due to its high fatality rate in later stages, timely treatment is strongly suggested once malignant cancer is detected. Compared to other standard treatment modalities (e.g., chemotherapy and radiotherapy) that might cause severe side effects,(Miller et al., 2019; Barazzuol et al., 2020; Qing-Yu Zhang, 2018)radiofrequency ablation (RFA) is regarded as a promising minimally invasive cancer treatment with relatively gentle side effects and has been widely used for years, ${ }^{(\mathrm{Ni} \text { et al., } 2005 \text {; Kok et al., 2020) }}$ particularly for small sized ones (radius smaller than $3 \mathrm{~cm}$ ). During RFA treatments, a key point lies in the generation of ideal RFA lesions that cover specific areas/volumes where target tissues exist. For large areal and highly efficient ablations, intensive efforts have been made for the development of new devices, such as expandable multi-tined electrodes and perfusion elec-

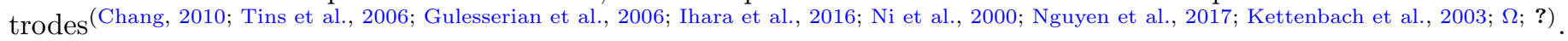

Although these devices can effectively ablate the target tissue either by increasing the surficial area of electrode (e.g., expandable multi-tined electrodes) or by cooling the overheated surface of electrode (e.g., perfusion electrode), the shape of RFA lesions are almost same and cannot be adjusted for target tissues that are irregularly shaped. (Chen et al., 2017; Vivek Kumar Singh, 2020; Golston et al., 1992; Mazurowski et al., 2017) They usually end up excessively ablating and often brings excessively irreversible damage to the organs' functions. Therefore, in the clinical practice, conformal RFA lesions is desired since it can shorten the time that patients take to recover and improve their live quality, especially for the organs, e.g., kidney, that have limited capability to regenerate after injury. (Chou et al., 2014)

To satisfy the diverse morphologic demanded in RFA lesions, several groups proposed various designs. Among them, the most influential strategy is to utilize multiple 
electrodes. (Takaki et al., 2013; Laeseke et al., 2006; Ritz et al., 2006; Peng et al., 2011) The multiple electrodes strategy, which distinguishes from multi-tined electrode strategy, simultaneously insert several electrodes into the body, and a pre-programmed alternative current is then used to energize the electrodes. The deployment of these electrodes can be delicately designed to better conform to the target tissues and avoid some unnecessary injuries of other organs. However, the classic single polar electrode, as well as the bipolar electrode, usually leads to multiple insertions into the body and increase the pain of patients. (Takaki et al., 2013; Laeseke et al., 2006; Ritz et al., 2006; Peng et al., 2011) What's more, the precise deployment of these electrodes is complex and the electrodes have to be supported by other tools to ensure the correct relative position. (Ritz et al., 2006; Peng et al., 2011) Hence, an operationally eased RFA device is urgently needed to achieve on-demand conformal ablation of diverse shaped target tissues in a highly efficient way.

Herein, we present an individually-controlled multi-tined expandable electrode for on-demand conformal RFA of target tissues, Figure 2A. The independent tunability of active cannula and pre-curved stylet inside and their following interactions in each sub-electrode contributes to the shape morphing of our electrode. These sub-electrodes are individually-controlled for two major advantages. On the one hand, the trajectory of individual sub-electrodes can be predicted for ablation of target tissues. On the other hand, combined with energy control, diverse sizes and shapes of RFA lesions can also be achieved by combining different electrode shapes, Figure 2B. Such advantages have been verified in later ex vivo experiments of irregular RFA lesions. Compared with the commercial electrodes, our electrode can change the shape of RFA lesion and ablate the target tissue on demand.

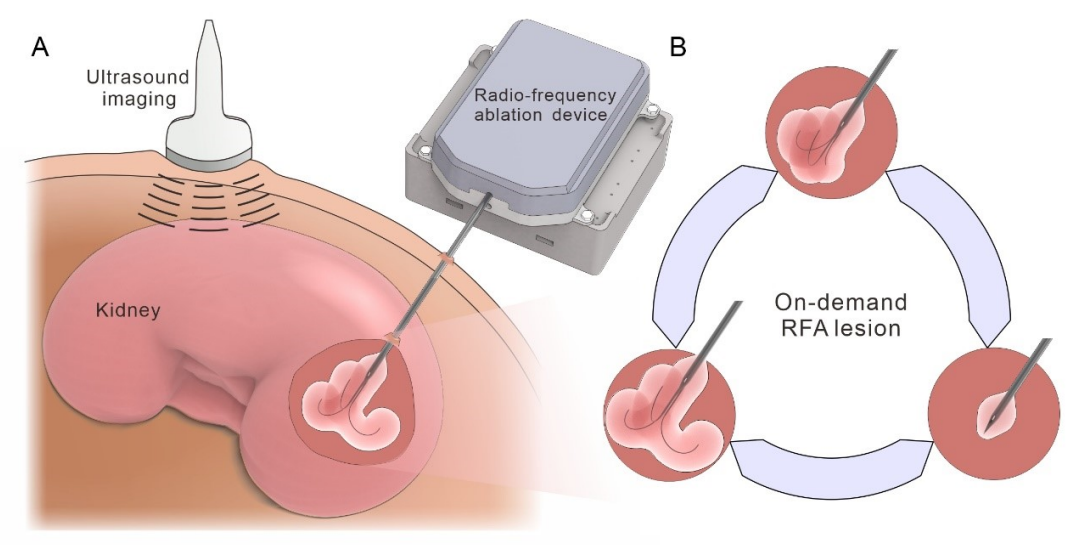

Figure 2: On-demand conformal ablation of proposed RFA device. (A) The concept of ablating the target tissue in-vivo under the guidance of an ultrasound imaging. (B) Flexibly tuning the RFA lesion by individually control the shape of each electrode.

\section{Results \& Discussion}

According to previous research, (Trujillo and Berjano, 2013) RFA lesions are associated with biophysical properties of tissues and will conform to the shape of electrode. To match the morphologic appearance (diverse ablation volumes in different 3D shapes) of RFA lesions and target tumor, and thus minimize unnecessary injury of normal cells, one of the most effective approaches is to properly design the electrode. (Zhang et al., 2016) As shown in Figure 3A, a flexible pre-curved stylet and a relative stiff coaxial cannula are designed to tune the final expanded-out electrode shape by adjusting the relative axial position of them. Three individuallycontrolled sub-electrodes are integrated in one electrode for the rational control of energy output, and hence three-dimensional on-demand conformal ablation can be achieved in an efficient way. 

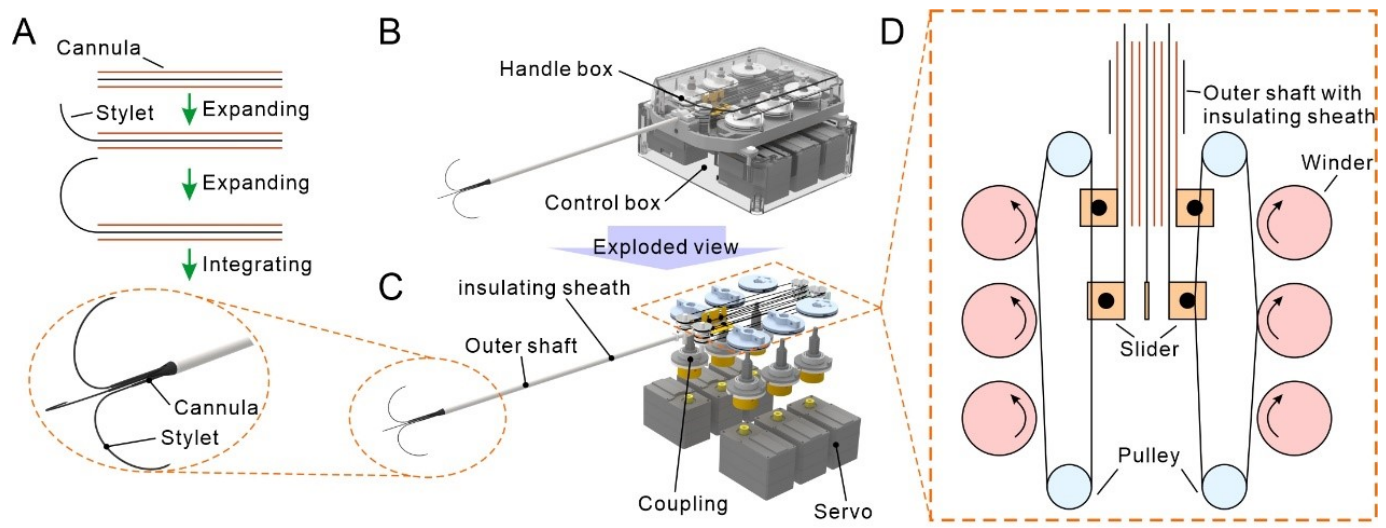

Figure 3: The structure of radio-frequency ablation device. (A) Design of expandable sub-electrode. (B) Overall view of the ablation device. (C) Exploded view of the ablation device. (D) Schematic of the multitined electrode actuation.

\section{Design of RFA device}

As shown in Figure 3B, the individual control of these sub-electrodes and their stylets and cannulas requires a proper design of RFA device. Sub-electrodes can be withdrawn/expanded inside the outer shaft of electrode, and the head of electrode is designed to be a cone for better tissue insertion. The outer shaft is covered with an insulating polymer sheath for the electric insulation. To avoid possible cross-infection, the invasive part of surgical tools are often designed as single use. However, as the control and driven module are essential parts in our electrode design, our proposed RFA device consists of a disposable handle box where electrode is integrated and a reusable control box (photos in Figure 4), which are both modularized components for convenient assembly and re-assembly. In the handle box, each of the stylets and cannulas is fixed to a sider and they only have one degree of freedom, Figure 3C-D. Steel wires wound on the winders connect to the slider for tendon-driven control of these stylets and cannulas. The winders are the interface of handle box to connect the control box. Six servos in the control box actuate their winders accordingly via an array of couplings. These servos are pre-programmed to follow real-time instructions for various motions. Our device is designed to be compatible with a commercial RF generator and the detailed operation method is elaborated in Experimental section text and Figure 5.

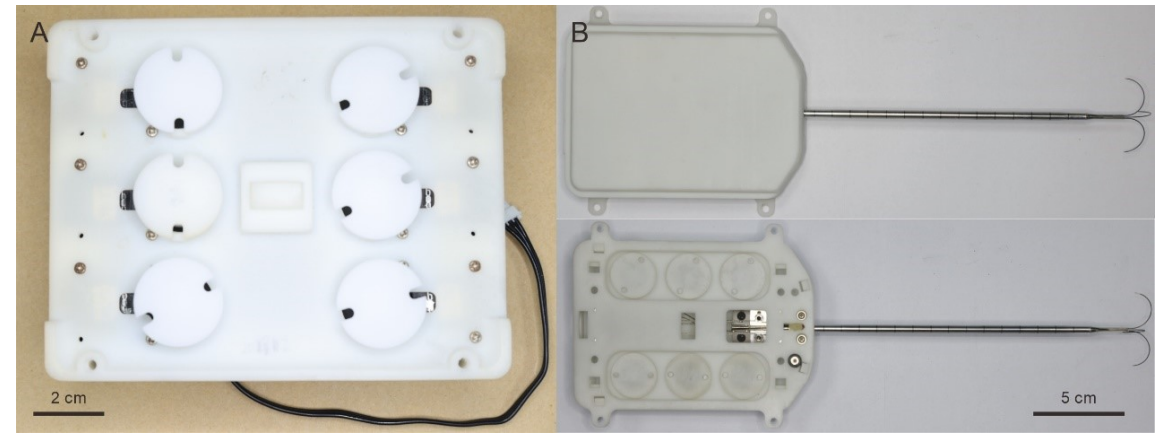

Figure 4: The Photos of RFA device. (A) The picture of control box. (B) The pictures of handle box. 


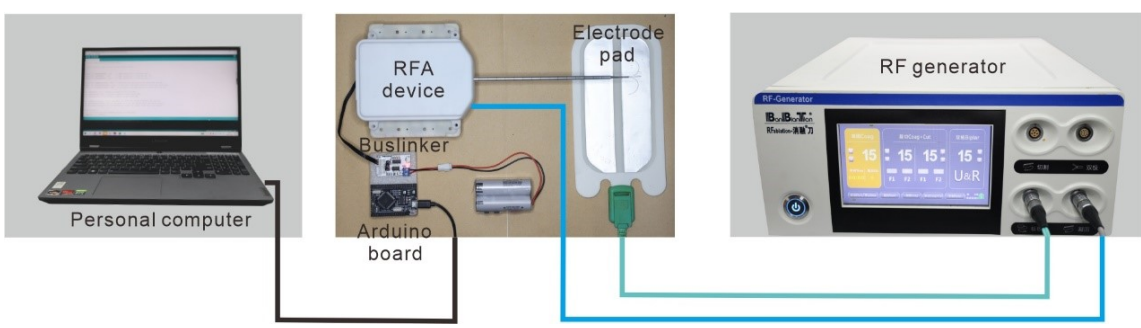

Figure 5: The whole radiofrequency ablation system and its connections.

\section{Shape morphing of sub-electrodes by stylet and cannula tuning}

Owing to the pre-curved shape and the bevel tip of the stylet (the detailed structure of stylet and cannula depicted in Figure 6), the shape of sub-electrode can be tuned on demand by adjusting the relative axial position of expanded stylet and cannula. As illustrated in Figure 7A, the expanding of a sub-electrode can be divided into three morphing states that are featured by the length of stylet remained in the cannula (it has to be noticed that the expanded length of stylet is no less than that of cannula):

State 1, the stylet is expanding with the cannula. In this state, the expanded length of stylet equals to that of cannula. The cannula is somehow deflected by the pre-curved stylet and thus create a slightly deflected angle of the sub-electrode. (The cannula is illustrated to be fully expanded in Figure 7A.)

State 2, the stylet is expanded out of the cannula and the expanded part of cannula is occupied by the pre-curved part of stylet. In this state, the pre-curved part of stylet is not fully expanded so that the straight part of stylet do not enter into the cannula. For a certain expanded length of cannula, the deflected angle keeps almost unchanged in this state and a circle can be used to fit the profile of the pre-curved part of stylet. (Gerboni et al., 2017; Adebar et al., 2016) (Apart from stylet, expanded length of cannula is also tunable, and the cannula is illustrated to be fully expanded in Figure 7A.)

State 3, the stylet is expanded out of the cannula and the straight part of stylet exists in the cannula. For a certain expanded length of cannula, the deflected angle in this state is subsequently smaller than that in state 1 and 2. (Similarly, expanded length of cannula is also tunable in this state, and the cannula is illustrated to be fully expanded in Figure 7A.)

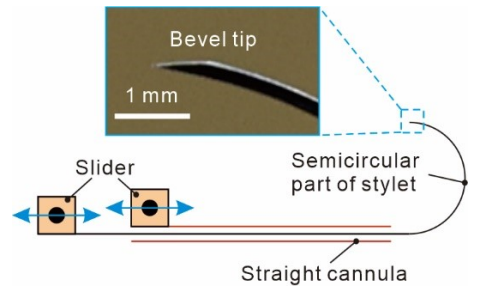

Figure 6: The detailed structure of a sub-electrode.

All of these three states of a sub-electrode can be observed in the air, Figure 7B. Of course, in the tissue, the damping force of nearby tissues can influence the shape as well during the expanding process. Hence, the influence of biological tissue is still worth being investigated, Figure 7C (original photos in Figure 8). Due to the existence of tissue, the deflection of sub-electrode is weakened and results in relatively smaller magnitude of deflection angle in morphing state 1, Figure 7D. In state 2, the stylet is expanded out of the cannula and begin to contact with the tissue. As mentioned above, the deflected angle is almost unchanged and the radius of curved stylet also seldom changes. In state 3 , not only the recovery of deflected cannula 

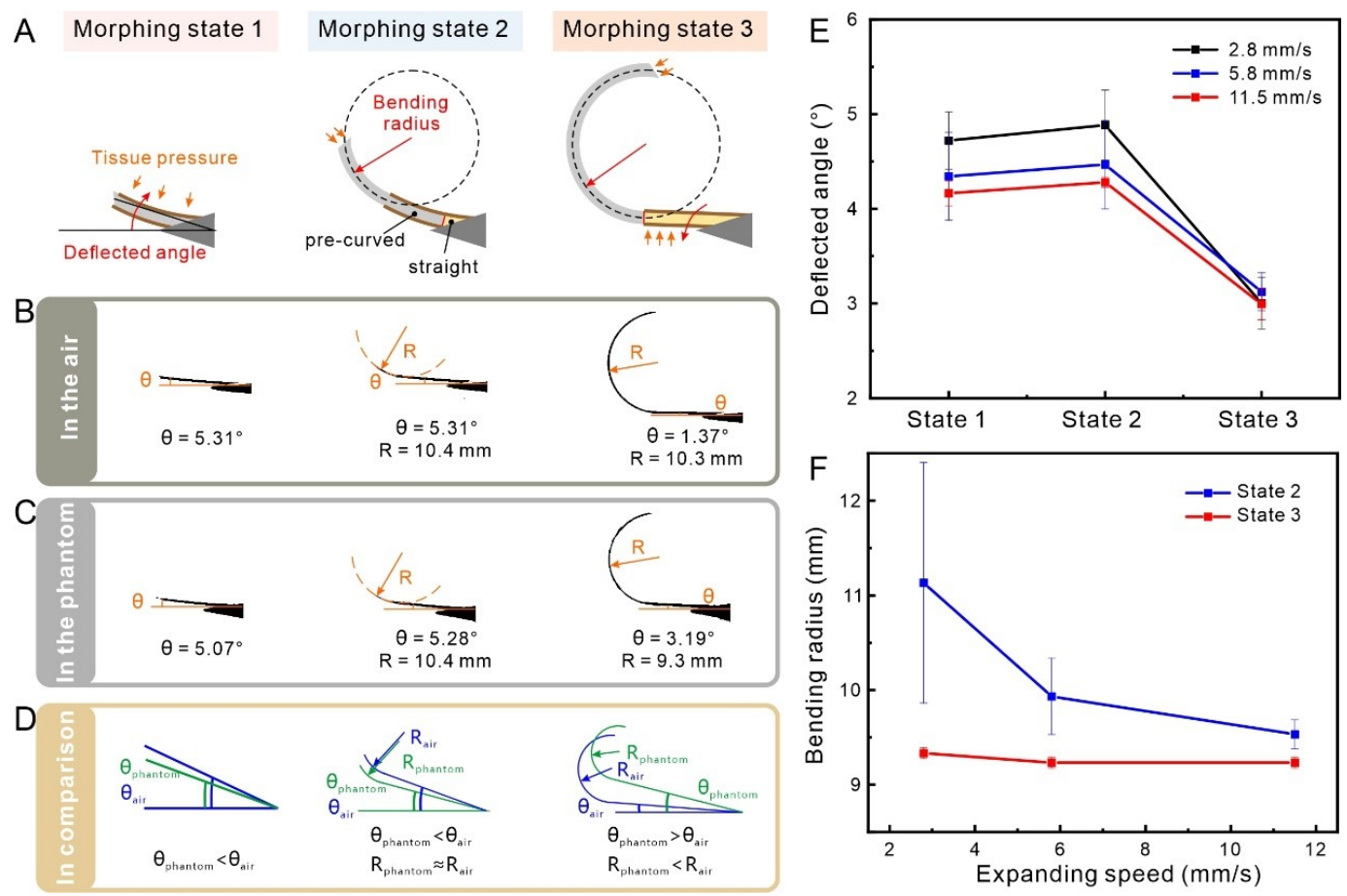

Figure 7: The shape morphing of sub-electrode and its interaction with tissue phantoms. (A) Shape morphing principle of sub-electrode. The cannulas are fully expanded in each morphing state here. The enhanced images of a sub-electrode in the (B) air and in the (C) phantom, marked with deflected angles and bending radii. (D) The comparison of the shape parameters of sub-electrode in the air and in the phantom. (E) The change of deflected angle in different morphing states. (F) The change of bending radius with the increment of expanding speed.

is damped by the tissue, but also the bevel tip of stylet is further affected by the tissue. Therefore, in this state, the deflected angle of cannula in tissue phantom is larger than that in air, and the bending radius of stylet in the tissue phantom is smaller than that in air.

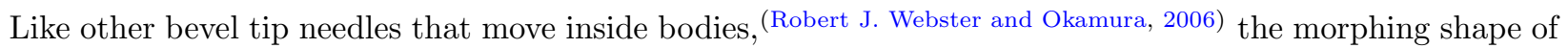
our active-cannula-based pre-curved stylet is also coupled to its insertion speed. By carefully analyzing the obtained data, it is found that the higher insertion speed is, the larger force is exerted on the sub-electrode by the tissue phantom and thus yields a lower deflection angle in a reasonable range, Figure 7E. Meanwhile, the impact of speed-relevant force is more obvious in state 2 than that in state 3 , and the higher the speed is, the stronger the impact is, Figure 7F. The quantitively analysis of the sub-electrode provides a useful guidance for the shape morphing and trajectory control of our electrode in the tissue phantom.

\section{Hosted file}

movieS1.mp4 available at https://authorea.com/users/451898/articles/550124-an-individuallycontrolled-multi-tined-expandable-electrode-using-active-cannula-based-shape-morphing-

for-on-demand-conformal-radiofrequency-ablation-lesions

\section{Trajectory control in tissue phantoms}

To ablate the target tissue on demand, the accurate trajectory of the electrode is necessary. Here, to reach for certain target points, the regions of insertion positions are quantitively analyzed using the measured 

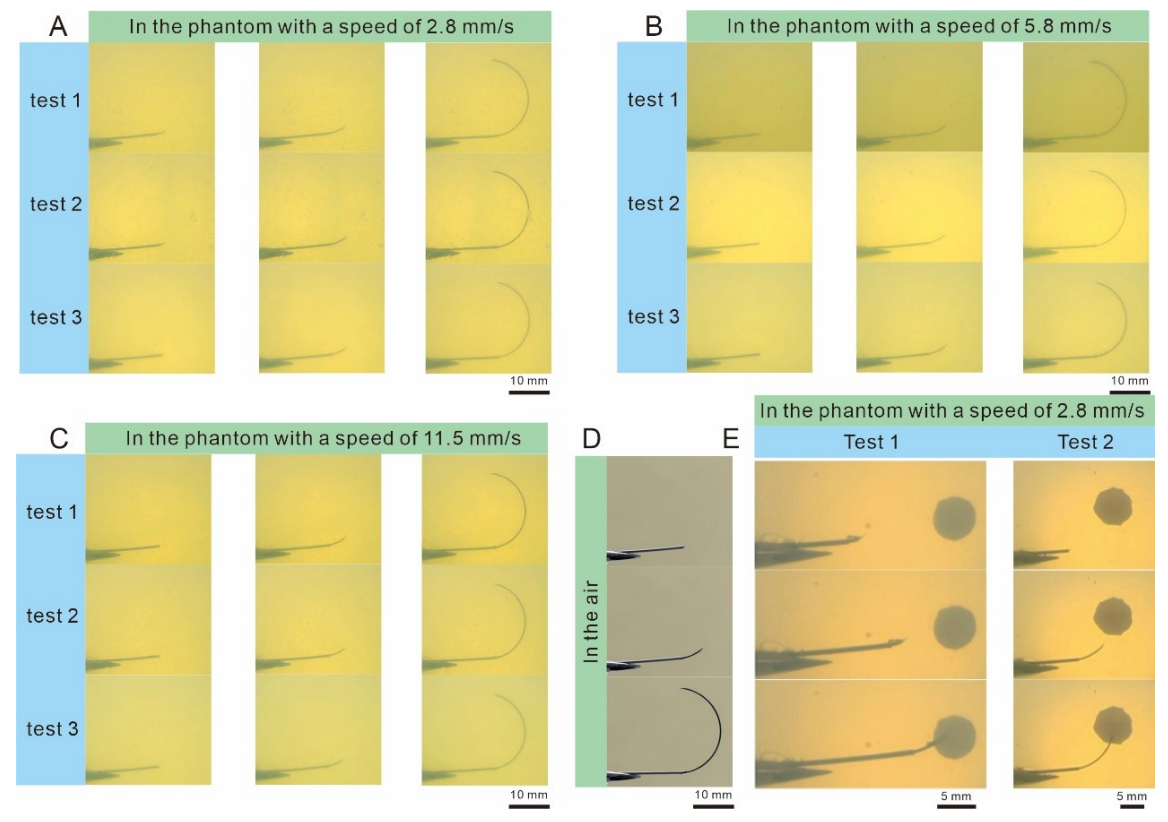

Figure 8: The snapshots of an expanded sub-electrode: (A-C) in the phantoms with a different insertion speed, and (D) in the air with a speed of $2.8 \mathrm{~mm} / \mathrm{s}$; (E) a sub-electrode expanding for a target (black circle) in tissue phantom.

data in Figure 7. As shown in Figure 9, the regions of insertion positions are plotted and comparisons are shown with the total region. The insertion angle of our electrode is fixed to mimic the practical condition that might happen during an RFA. Owing to the shape tunability of our sub-electrode, the electrode tip can successfully reach the target within a wide range of insertion positions. The insertion position region is becoming smaller and moving closer to the target point with the increase of expanding speed. Such a speed-relevant characteristic might be utilized to compensate the insertion error. What's more, the plenty choices of insertion position will help to avoid inserting into vital vessels or hurting important functional parts in the body and further reduce the possible risks that might cause during the ablation. The accurate trajectory to a certain target is further verified as shown in Figure 9B-C. Given a certain expanding speed, our electrode is able to reach the target point at different insertion positions.

\section{Morphologic Characterization of RFA Lesions}

By means of finite element analysis, the mechanism of on-demand conformal RFA is further investigated. Since the morphologic appearance of RFA lesion mainly contains size (or ablated volume) and shape, these two aspects are considered in our performance evaluation. As illustrated in Figure 10A, the heat will accumulate in the surrounding tissues and thus cause overheat of tissues, which prevent further enlargement of RFA lesion. As further revealed in Figure 10B, rapid impedance increase occurs with the slowdown of the increasing rate of ablated volume. The ablated area starts from the tip of the electrode and finally forms a shape that is similar to the shape of electrode (insets in Figure 10B and Figure 11). Owing to such an effect, we are able to adjust the expanded length of the electrode to adapt diverse shapes of RFA lesion and achieve different volumes of RFA lesion, Figure 10C.

Apart from ablation time and expanded length, the applied voltage/power also plays an important part in the determination of RFA lesions. Within our investigated range, the ablated volume peaks at around $12 \mathrm{~V}$ and it overpasses 4 times of the volume at $8 \mathrm{~V}$, Figure 10D. In fact, low voltage/power heat the nearby tissue 

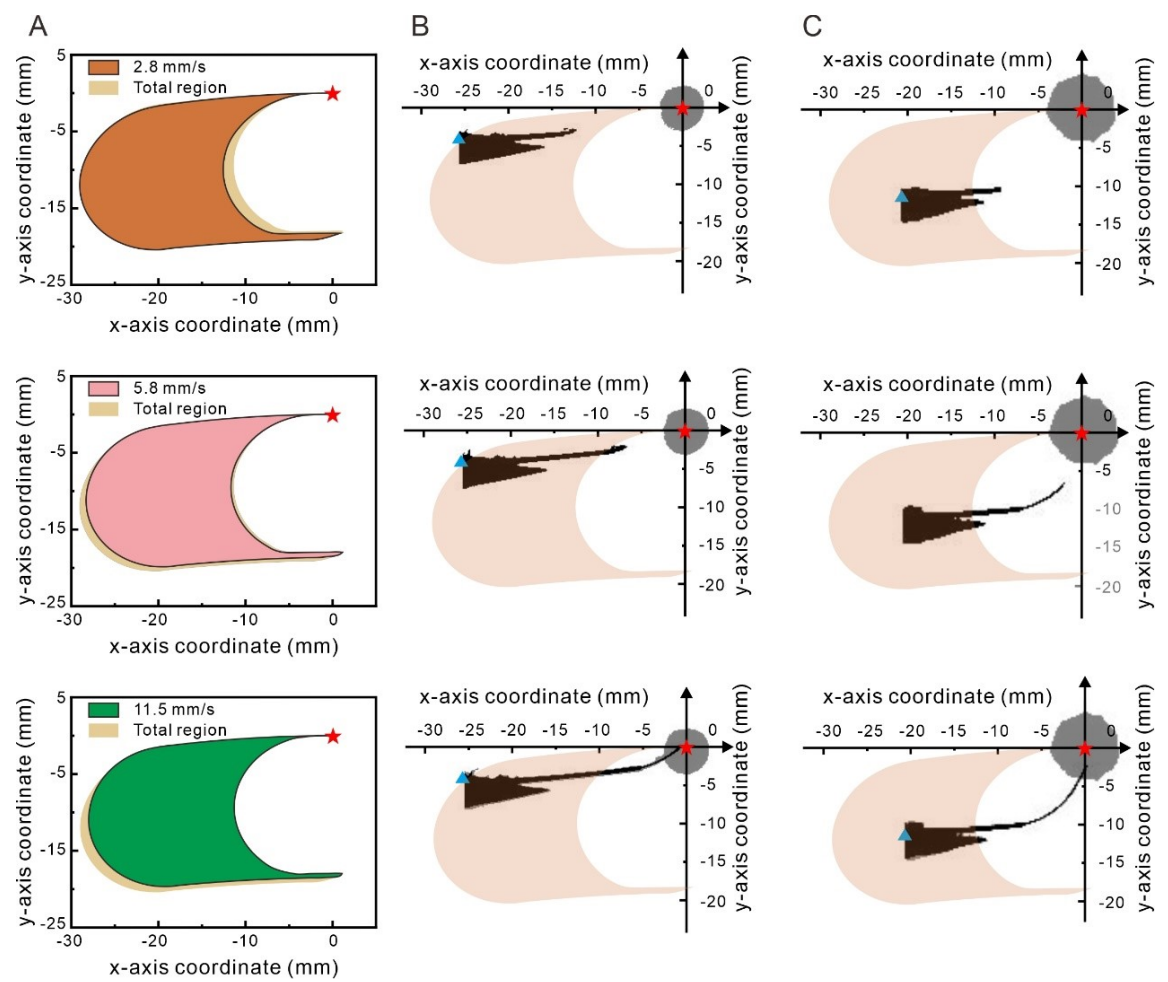

Figure 9: The insertion position region of a sub-electrode and its verification. (A) The insertion position region for a target of a sub-electrode under different expanding speed. The total region is the combination of regions under different expanding speed (B and C) The verification of the reach of the target at different insertion position. The expanding speed of the electrode is kept $2.8 \mathrm{~mm} / \mathrm{s}$.

slowly and the overheating of tissue doesn't occur within the investigated range (curves of $8 \mathrm{~V}$ and $10 \mathrm{~V}$ in Figure 12). In contrast, high voltage/power heat the tissue rapidly, and the higher the voltage/power is, the earlier overheating occurs (curves of $12 \mathrm{~V}-18 \mathrm{~V}$ in Figure 12). Therefore, it's also important to choose a proper voltage for RFA planning.

As our sub-electrodes are individually-controlled, any sub-electrodes can be expanded on demand to enlarge the ablated volume of electrode, Figure 10E. Moreover, by expanding all of the three stylets and cannulas differently, the electrode will have distinct shape and volume of RFA lesions, Figure 10F.

To sum it up, the RFA lesion of our electrode is influenced by three hierarchical factors. The rationally controlled ablation time and applied voltage/power offer energy to the electrode. Three sub-electrode can individually expand to conform to target tissues and share delivered energy. The stylet and cannula in each sub-electrode further morph by adjusting their relative position and hence expanded length. Thanks to these factors, our electrode can thus have high potential to meet diverse demands of tumor treatments with various morphologic appearances.

\section{Ex vivo On-demand Conformal RFA}

To further verify our proposed device, several ex vivo experiments are conducted on fresh porcine kidneys. A commercial ablation electrode (photo in Figure 13) is also used as a reference. Both electrodes are used to ablate a target tissue with same ablation parameters (ablation time, insertion depth and ablation power). To visualize and evaluate the RFA lesions, the ablated kidneys are sliced carefully at the surface where 

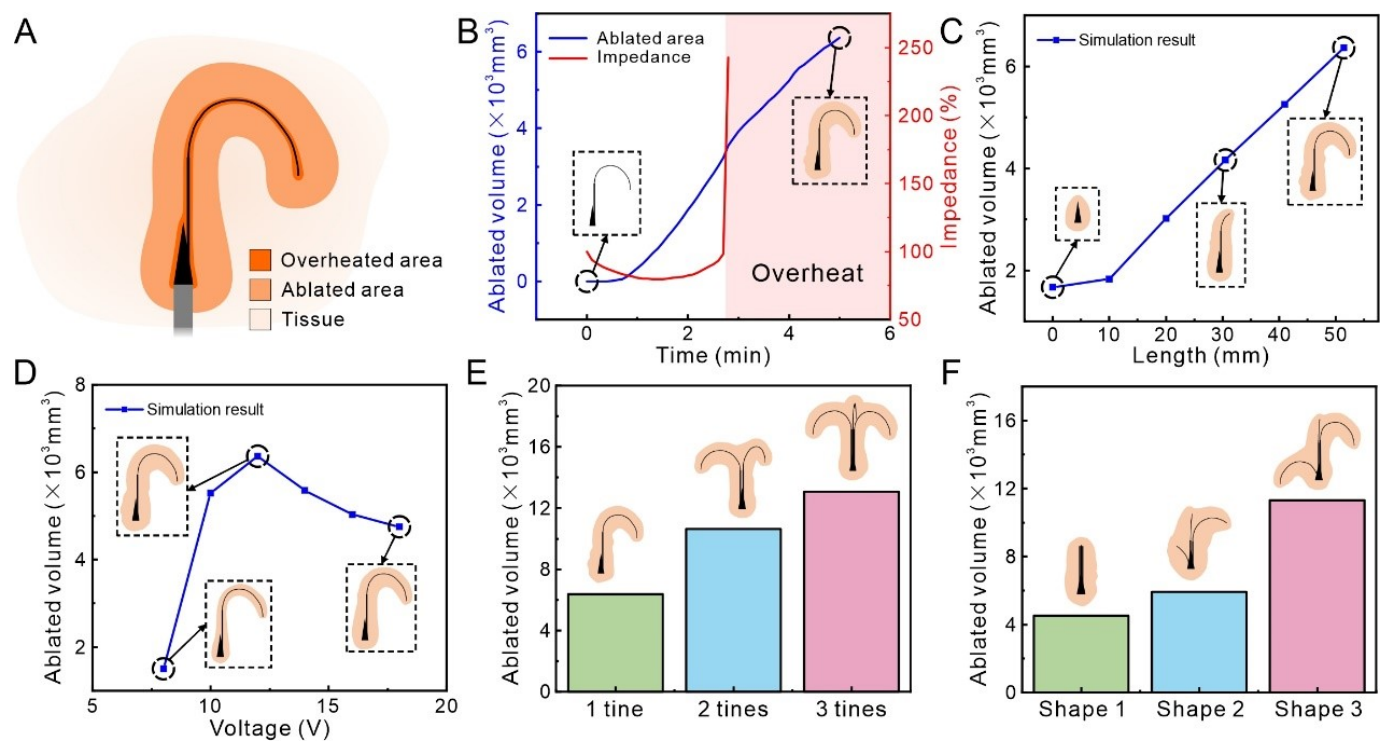

Figure 10: Morphologic characterization of RFA lesions. (A) Conformal ablation of our electrode. (B) Simulated ablated volume and its corresponding impedance under different ablation time (the stylet and cannula are fully expanded). (C) Simulation results of the relation between ablated volume and the expanded length of electrode. (D) Simulation results of the relation between ablated volume and the applied voltage. (E) Influence of number of expanded tines to ablated volumes and shapes. (F) Impact of shapes of tines on the ablated volumes and shapes.

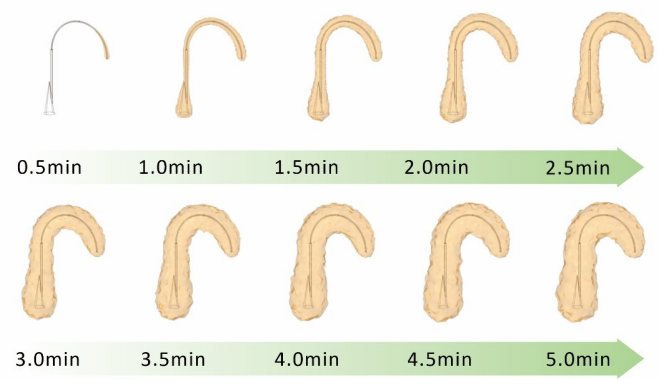

Figure 11: The evolution of simulated RFA lesion along time (0-5 min).

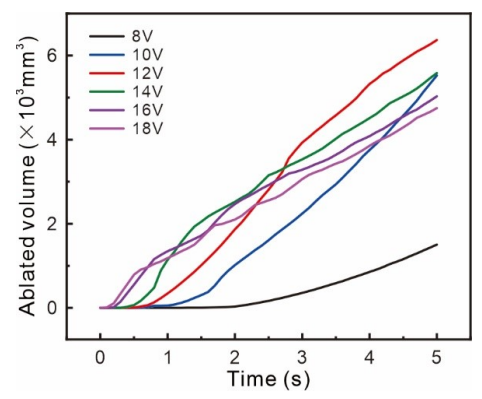

Figure 12: The evolution of ablated volume under different applied voltages along time (0-5 min). 
the (sub)electrode is. As illustrated in Figure 14A (i and ii), small RFA lesions with nearly circular and elliptic sectional contours can be easily observed and the sectional areas of RFA lesions are roughly the same. However, the stiffer commercial electrode is impossible to steer inside the tissue, which make it hard to conform to a curved sectional shape, Figure 14A (iii). In contrast, our pre-curved electrode can conform well and ablate a curved sectional shape that is almost identical with the target tissue. Additionally, such an on-demand conformal ablation is also applicable for other tissues, e.g. a porcine liver in Figure 15.

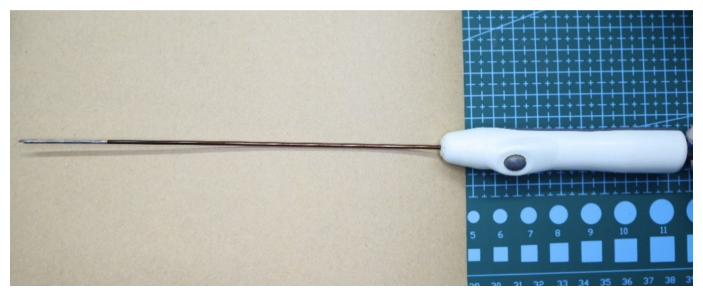

Figure 13: The photo of a commercial available electrode.

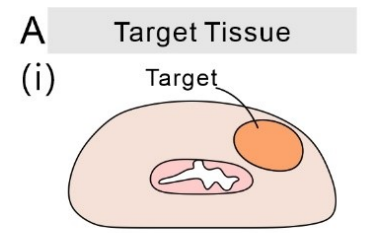

(ii)

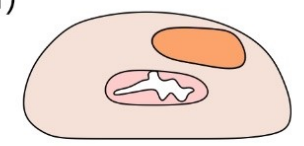

(iii)

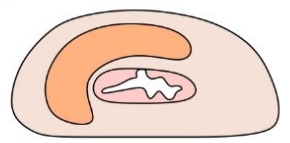

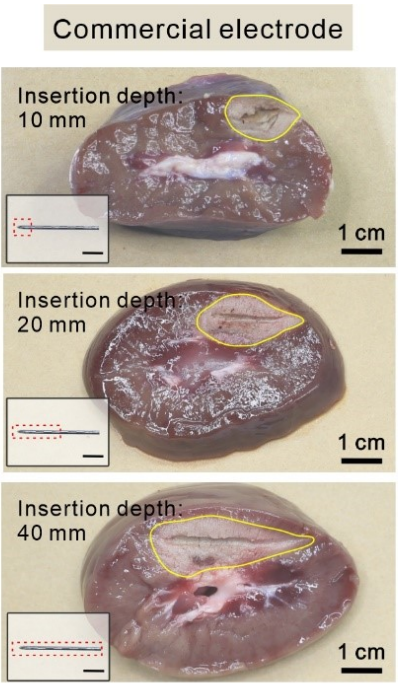
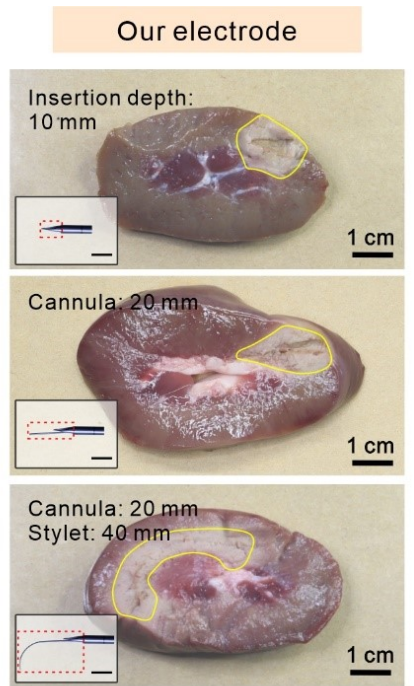

B

\section{Cannula length changes, stylet remain constant}
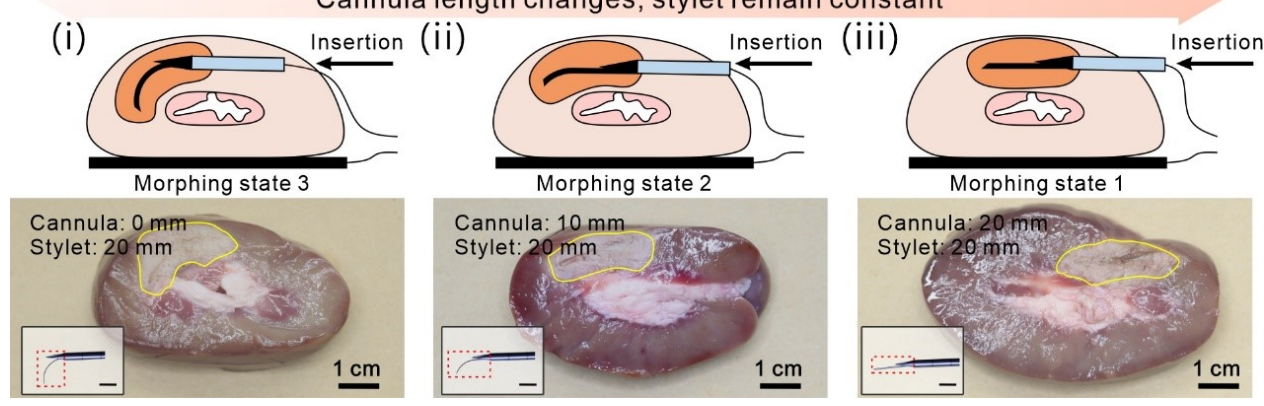

Figure 14: Ex vivo RFA of porcine kidneys. (A) The comparison of a commercial RFA electrode and our proposed electrode ablating several target tissues. (B) The morphologic change of RFA lesions with the shape change of expanded electrode of which the length and insertion angle are kept constant.

Given the same expanded length but different morphing states of the sub-electrode (which means the length of stylet keeps constant), different RFA lesions with the same sectional area can be achieved by the change 

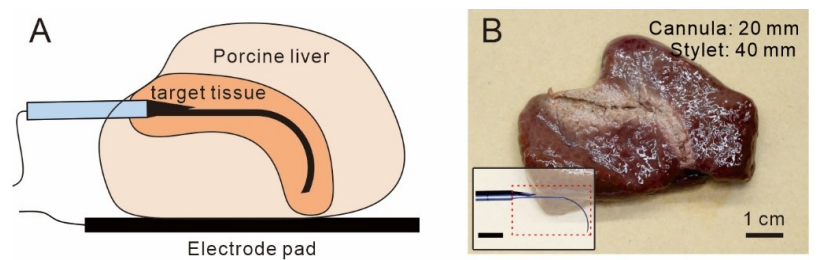

Figure 15: Target tissue ablation in a porcine liver using our electrode. (A) The schematic illustration. (B) The sectional view after ablation.

of cannula length, Figure 14B, and it indicates that our electrode is able to ablate malignant tumors with the same size but different shapes. In addition, the insertion pose (which means the angle and position) of electrode are restricted during the experiment to mimic the actual situation for the safety of operation, and RFA lesions with distinct positions are still achieved.

\section{Hosted file}

movieS2.mp4 available at https://authorea.com/users/451898/articles/550124-an-individuallycontrolled-multi-tined-expandable-electrode-using-active-cannula-based-shape-morphingfor-on-demand-conformal-radiofrequency-ablation-lesions

\section{Conclusion}

We propose an individually-controlled multi-tined expandable electrode for the on-demand RFA lesions. The interaction between pre-curved flexible stylet and relatively stiff cannula produces various shapes of our sub-electrodes. By tuning the expanded length and expanding speed of the stylets and cannulas, diverse trajectories can be realized on demand. Along with a rationally applied energy, three individually-controlled sub-electrodes can achieve three-dimensional on-demand conformal ablation efficiently. The ex vivo RFA experiments of porcine kidney and its comparison with a commercial electrode further demonstrate the conformability of our RFA lesions. Our electrode exhibits the potential to conformally ablate in vivo target tissue with various morphologic appearances on demand.

\section{Experimental Section/Methods}

\section{Operation of the radiofrequency ablation system based on our RFA device}

As illustrated in Figure 5, the RFA device and an electrode pad is connected to the RF generator for ablation. The servos in control box of our RFA device is actuated by Buslinker, which is powered by a battery pack. An Arduino board (Mega 2560 Pro) is used to receive messages from a personal computer, and send commands to the Buslinker and finally control the servos.

\section{Trajectory fitting of the sub-electrode}

Two hypotheses are assumed in this model:

1. The trajectory of sub-electrode is considered to be constructed by several segments of arcs, which is widely adopted in other works(Swaney et al., 2013; Adebar et al., 2016; Gerboni et al., 2017).

2. To simplify the model, the shape of stylet and cannula is assumed to change linearly during the morphing, and the fully expanded state (the stylet and cannula are both maximally expanded) of each morphing state is utilized for fitting. 
Based on the hypotheses mentioned above, the structure parameters can be expressed as follows:

$\theta=\frac{\theta_{1} l_{c}}{L_{c}}, l_{s}=l_{c}$

$\theta=\left[\theta_{1}\left(\frac{L_{p s}-L_{s}}{L_{p s}-l_{c}}\right)+\theta_{2}\left(\frac{L_{s}-L_{c}}{L_{p s}-l_{c}}\right)\right] \frac{l_{c}}{L_{c}}, l_{s} \leq L_{p s}$

$\theta=\left[\theta_{2}\left(\frac{L_{c}+L_{p s}-L_{s}}{l_{c}}\right)+\theta_{3}\left(\frac{L_{s}-L_{p s}}{l_{c}}\right)\right] \frac{l_{c}}{L_{c}}, l_{s}>L_{p s}$

$r=r_{1}, l_{s}-l_{c} \leq L_{p s}-L_{c}$

$r=r_{1}\left(\frac{L_{c}+L_{p s}-L_{s}}{L_{c}}\right)+r_{2}\left(\frac{L_{s}-L_{c}}{L_{c}}\right), l_{s}-l_{c}>L_{p s}-L_{c}$

where $\theta$ and $r$ are the deflected angle of cannula and bending radius, respectively. $\theta_{1}, \theta_{2}$ and $\theta_{3}$ are the measured deflected angle of cannula at the end of each morphing state. $r_{1}$ and $r_{2}$ are the measured bending angle of stylet at the end of each morphing state. $l_{c}$ and $l_{s}$ are the expanded length of cannula and stylet, respectively. $L_{c}$ and $L_{p s}$ are the maximal expanded length of cannula $(20 \mathrm{~mm})$ and the length of pre-curved part of stylet $(32 \mathrm{~mm})$, respectively.

The $l_{c}$ and $l_{s}$ in equation (1) - (5) only need to satisfy the following criteria:

$l_{c} \leq l_{s}$

$l_{c}+L_{p s} \geq l_{s}$

With equations (1)-(7), Figure 9 can be obtained accordingly.

\section{Other Experimental Methods}

Preparation of Tissue Phantom: As a widely used tissue phantom, (Solazzo et al., 2005) agar (Shanghai Regal Biology Technology Co., Ltd, China) was adopted in this paper. $12 \mathrm{~g}$ agar as well as $8 \mathrm{~g} \mathrm{NaCl}$ (Sinopharm Chemical Reagent Co., Ltd, China) was added in the $800 \mathrm{~g}$ boiled water and stirred for 12 min to be fully dissolved. Then the solution was poured in a mold and cooled for 1 hour to be cured and finally the tissue phantom was made.

Preparation of Tissue Phantom with an Opaque Target: The evenly mixed solution of agar was first poured in a mold and cured for 8 min until there is a thin layer of film formed on the surface. Then a droplet of ink was dropped on the film and waited for 5 min until the ink was dried. Finally, the mixed solution was again poured in the mold and cooled for 1 hour for curation. (The tissue phantoms with an Opaque target were molded for the visualization of our target in Figure 9.)

Equipment: An RF generator (BanBianTian, China) worked at a power of $15 \mathrm{~W}$ in the experiments and the frequency of $\mathrm{AC}$ is nominally $550 \pm 40 \mathrm{kHz}$. A magnetic mixer (HS 7, IKA, Germany) was used for the mixing and heating of the solution.

Image processing: The captured colored pictures are imported into MATLAB 2019a to transform to grayscale images. After a threshold is selected for each sets of images according to the brightness of the pictures, these grayscales images are then transformed to black and white images.

Finite Elements Analysis: COMSOL Multiphysics v5.6 (Stockholm, Sweden) was used for the radiofrequency ablation analysis. All the electromagnetic and thermal properties of the tissues are provided by COMSOL Multiphysics. For the simplicity of our model, blood perfusion and heat flux with the external environment are not considered, and only the conductive cone head and sub-electrodes are considered in this model. Arrhenius kinetics model was used to assess the damage of tissues. All the insets in Figure 10 are reprocessed for better visualization, the original pictures exported from COMSOL Multiphysics are illustrated in Figure 16. 
A
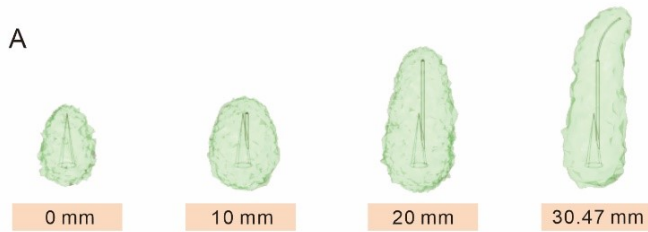

$30.47 \mathrm{~mm}$

B

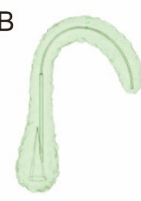

$8 \mathrm{~V}$

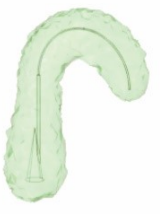

$10 \mathrm{~V}$

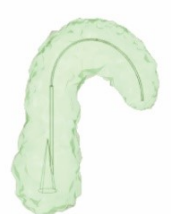

$12 \mathrm{~V}$

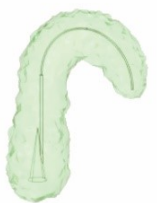

$14 \mathrm{~V}$

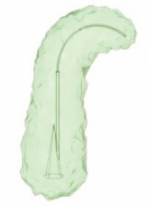

$40.93 \mathrm{~mm}$

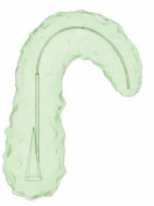

$16 \mathrm{~V}$

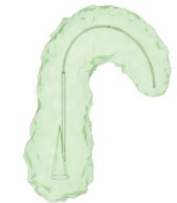

$51.42 \mathrm{~mm}$

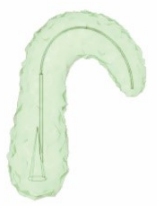

$18 \mathrm{~V}$

C

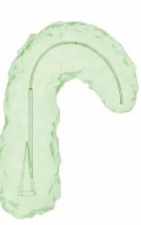

1 tin
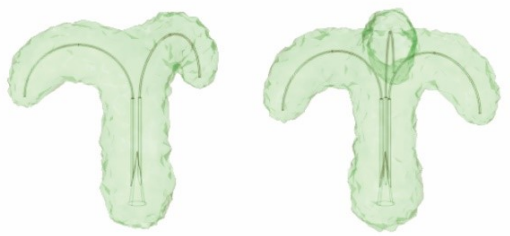

2 tins

3 tins

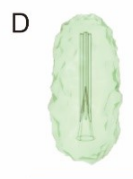

Shape 1

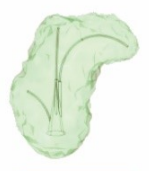

Shape 2

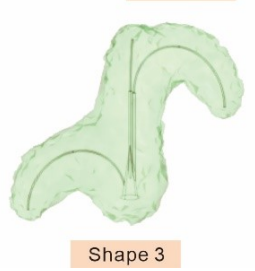

Figure 16: Original picture of the insets in Figure 10 exported from COMSOL Multiphysics. The change of RFA lesion with the (A) increment of expanded length, (B) growth of applied voltage, (C) the number of expanded electrodes, (D) the change of RFA lesion with different shape of sub-electrodes.

Porcine Kidneys and Livers Preparation: the experiments were carried out in accordance with the guidelines issued by the Ethical Committee of Huazhong University of Science and Technology for the National Key R\&D program of China (Grant No. 2017YFB1303100). 


\section{Acknowledgements}

This work was financially supported by National Key R\&D program of China (Grant No. 2017YFB 130103). Zhiping Chai and Liangxiong Lyu contribute equally in this work.

\section{Conflict of interest}

The authors declare no conflict of interest.

\section{References}

TK Adebar, JD Greer, PF Laeseke, GL Hwang, and AM Okamura. Methods for Improving the Curvature of Steerable Needles in Biological Tissue. IEEE Trans Biomed Eng, 63:1167-77, Jun 2016.

L Barazzuol, RP Coppes, and Luijk P van. Prevention and treatment of radiotherapy-induced side effects. Mol Oncol, 14:1538-1554, Jul 2020.

F Bray, M Laversanne, E Weiderpass, and I Soerjomataram. The ever-increasing importance of cancer as a leading cause of premature death worldwide. Cancer, 127:3029-3030, Aug 2021.

IA Chang. Considerations for thermal injury analysis for RF ablation devices. Open Biomed Eng J, 4:3-12, Feb 2010.

T Chen, PH Zhou, Y Chu, YQ Zhang, WF Chen, Y Ji, LQ Yao, and MD Xu. Long-term Outcomes of Submucosal Tunneling Endoscopic Resection for Upper Gastrointestinal Submucosal Tumors. Ann Surg, 265:363-369, Feb 2017.

YH Chou, SY Pan, CH Yang, and SL Lin. Stem cells and kidney regeneration. J Formos Med Assoc, 113: 201-9, Apr 2014.

G Gerboni, JD Greer, PF Laeseke, GL Hwang, and AM Okamura. Highly Articulated Robotic Needle Achieves Distributed Ablation of Liver Tissue. IEEE Robot Autom Lett, 2:1367-1374, Jul 2017.

JE Golston, WV Stoecker, RH Moss, and IP Dhillon. Automatic detection of irregular borders in melanoma and other skin tumors. Comput Med Imaging Graph, 16:199-203, May 1992.

T Gulesserian, AH Mahnken, R Schernthaner, M Memarsadeghi, M Weber, A Tacke, and J Kettenbach. Comparison of expandable electrodes in percutaneous radiofrequency ablation of renal cell carcinoma. Eur J Radiol, 59:133-9, Aug 2006.

H Ihara, H Gobara, T Hiraki, T Mitsuhashi, T Iguchi, H Fujiwara, Y Matsui, J Soh, S Toyooka, and S Kanazawa. Radiofrequency Ablation of Lung Tumors Using a Multitined Expandable Electrode: Impact of the Electrode Array Diameter on Local Tumor Progression. J Vasc Interv Radiol, 27:87-95, Jan 2016.

J Kettenbach, W Köstler, E Rücklinger, B Gustorff, M Hüpfl, F Wolf, K Peer, M Weigner, J Lammer, W Müller, and SN Goldberg. Percutaneous saline-enhanced radiofrequency ablation of unresectable hepatic tumors: initial experience in 26 patients. AJR Am J Roentgenol, 180:1537-45, Jun 2003.

HP Kok, ENK Cressman, W Ceelen, CL Brace, R Ivkov, H Grüll, Haar G Ter, P Wust, and J Crezee. Heating technology for malignant tumors: a review. Int J Hyperthermia, 37:711-741, 2020.

PF Laeseke, LA Sampson, D Haemmerich, CL Brace, JP Fine, TM Frey, TC 3rd Winter, and FT Jr Lee. Multiple-electrode radiofrequency ablation creates confluent areas of necrosis: in vivo porcine liver results. Radiology, 241:116-24, Oct 2006. 
MA Mazurowski, K Clark, NM Czarnek, P Shamsesfandabadi, KB Peters, and A Saha. Radiogenomics of lower-grade glioma: algorithmically-assessed tumor shape is associated with tumor genomic subtypes and patient outcomes in a multi-institutional study with The Cancer Genome Atlas data. J Neurooncol, 133: 27-35, May 2017.

KD Miller, L Nogueira, AB Mariotto, JH Rowland, KR Yabroff, CM Alfano, A Jemal, JL Kramer, and RL Siegel. Cancer treatment and survivorship statistics, 2019. CA Cancer J Clin, 69:363-385, Sep 2019.

DT Nguyen, EP Gerstenfeld, WS Tzou, PT Jurgens, L Zheng, J Schuller, M Zipse, and WH Sauer. Radiofrequency Ablation Using an Open Irrigated Electrode Cooled With Half-Normal Saline. JACC Clin Electrophysiol, 3:1103-1110, Oct 2017.

Y Ni, Y Miao, S Mulier, J Yu, AL Baert, and G Marchal. A novel cooled-wet electrode for radiofrequency ablation. Eur Radiol, 10:852-4, 2000.

Y Ni, S Mulier, Y Miao, L Michel, and G Marchal. A review of the general aspects of radiofrequency ablation. Abdom Imaging, 30:381-400, Jul 2005.

HL Ormel, der Schoot GGF van, NL Westerink, WJ Sluiter, JA Gietema, and AME Walenkamp. Selfmonitoring physical activity with a smartphone application in cancer patients: a randomized feasibility study (SMART-trial). Support Care Cancer, 26:3915-3923, Nov 2018.

ZW Peng, HH Liang, MS Chen, YJ Zhang, YQ Zhang, and WY Lau. Conformal radiofrequency ablation of hepatocellular carcinoma with a multi-pin bipolar system. J Surg Oncol, 103:69-74, Jan 2011.

Ke-Ke Jia Ling-Dong Kong Qing-Yu Zhang, Fei-Xuan Wang. Natural Product Interventions for Chemotherapy and Radiotherapy-Induced Side Effects. Frontiers in Pharmacology, 9, 2018.

JP Ritz, KS Lehmann, C Reissfelder, T Albrecht, B Frericks, U Zurbuchen, and HJ Buhr. Bipolar radiofrequency ablation of liver metastases during laparotomy. First clinical experiences with a new multipolar ablation concept. Int $J$ Colorectal Dis, 21:25-32, Jan 2006.

Jin Seob Kim-Noah J. Cowan-Gregory S. Chirikjian Robert J. Webster, III and Allison M. Okamura. Nonholonomic Modeling of Needle Steering. International Journal of Robotics Research, 25, 2006.

SA Solazzo, Z Liu, SM Lobo, M Ahmed, AU Hines-Peralta, RE Lenkinski, and SN Goldberg. Radiofrequency ablation: importance of background tissue electrical conductivity-an agar phantom and computer modeling study. Radiology, 236:495-502, Aug 2005.

H Sung, J Ferlay, RL Siegel, M Laversanne, I Soerjomataram, A Jemal, and F Bray. Global Cancer Statistics 2020: GLOBOCAN Estimates of Incidence and Mortality Worldwide for 36 Cancers in 185 Countries. $C A$ Cancer J Clin, 71:209-249, May 2021.

PJ Swaney, J Burgner, HB Gilbert, and RJ 3rd Webster. A flexure-based steerable needle: high curvature with reduced tissue damage. IEEE Trans Biomed Eng, 60:906-9, Apr 2013.

H Takaki, A Nakatsuka, J Uraki, T Yamanaka, M Fujimori, T Hasegawa, K Arima, Y Sugimura, and K Yamakado. Renal cell carcinoma: radiofrequency ablation with a multiple-electrode switching system-a phase II clinical study. Radiology, 267:285-92, Apr 2013.

B Tins, V Cassar-Pullicino, I McCall, P Cool, D Williams, and D Mangham. Radiofrequency ablation of chondroblastoma using a multi-tined expandable electrode system: initial results. Eur Radiol, 16:804-10, Apr 2006.

M Trujillo and E Berjano. Review of the mathematical functions used to model the temperature dependence of electrical and thermal conductivities of biological tissue in radiofrequency ablation. Int J Hyperthermia, 29:590-7, Sep 2013. 
Santiago Romani Farhan Akram-Nidhi Pandey Md. Mostafa Kamal Sarker Adel Saleh Meritxell Arenas Miguel Arquez Domenec Puig Jordina Torrents-Barrena Vivek Kumar Singh, Hatem A. Rashwan. Breast tumor segmentation and shape classification in mammograms using generative adversarial and convolutional neural network. Expert Systems With Applications, 139, 2020.

B Zhang, MA Moser, EM Zhang, Y Luo, C Liu, and W Zhang. A review of radiofrequency ablation: Large target tissue necrosis and mathematical modelling. Phys Med, 32:961-71, Aug 2016. 